\title{
REDES SOCIAIS E COMUNIDADES: DEFINIÇÕES, CLASSIFICAÇÕES E RELAÇÕES
}

Gilmara Teixeira Barcelos, PGIE/UFRGS, IF Fluminense Campus Campos-Centro gilmarab@iff.edu.br

Liliana Maria Passerino, PGIE/UFRGS

liliana@cinted.ufrgs.br

Patricia Alejandra Behar, NUTED-PGIE/UFRGS

patricia.behar@ufrgs.br

\section{RESUMO}

A criação de redes sociais e dos diversos tipos comunidades pode contribuir para a implementação de programas de formação de professores à medida que ampliam os espaços de interações. Nesse sentido, considera-se importante um aporte teórico que defina redes sociais e comunidades e, além disso, que apresente as relações que podem ser estabelecidas entre as mesmas. Este artigo apresenta a visão de vários autores sobre o referido tema. Para tanto, inicialmente, promove-se uma reflexão sobre redes sociais, definindo laços sociais e capital social. A seguir, são apresentadas classificações de comunidades, estabelecidas a partir de seus objetivos. Finalizando, caracterizam-se e exemplificam-se sites de redes sociais.

Palavras-chave: Redes sociais, Comunidades, Formação de professores

\section{SOCIAL COMMUNITIES AND NETWORKS: DEFINITIONS, CLASSIFICATIONS AND RELATIONS}

\section{ABSTRACT}

The creation of social networks and various types of communities can contribute to the implementation of training programs for teachers as they extend the space of interactions. Thus, it is important to define a theoretical foundation for social networks and communities, and present the relationships that may be established among them. This article presents the view of several authors on the subject. It first discusses social networks and conceptualizes social ties and social capital. Following, a classification of communities is given based on their established goals. The final part of the article presents characteristic and examples of social networking sites.

Keywords: Social Networks, Communities, Teacher education.

\section{Introdução}

Neste artigo discute-se o conceito de redes sociais e de alguns tipos de comunidades como aporte teórico para elaboração de um modelo de formação continuada para professores de Matemática.

No início do século XXI, ocorreu uma mudança na ênfase das teorias de aprendizagem, o foco passou da aprendizagem do indivíduo para a aprendizagem como parte de uma comunidade (Kilpatrick et al. 2003). Este fato é consequência, entre outros fatores, da crescente influência da teoria vygotskyana, a qual destaca a contribuição do outro para a aprendizagem de cada indivíduo, ou seja, caráter social e comunitário da aprendizagem. Além disso, nas sociedades atuais destaca-se a importância dos diferentes contextos de socialização, ou de prática, como geradores da aprendizagem (Illera, 2007). Nesse sentido, ressalta-se a relevância das redes sociais para o desenvolvimento pessoal e, consequentemente, para a formação de professores. Afinal, segundo Tardif e Raymond (2000) o saber docente é plural, uma vez que é obtido no contexto da história de vida, da carreira profissional. 
Outras expressões estão atreladas a redes sociais, a saber: comunidades, comunidades de aprendizagem (CA), comunidades de prática (CP), comunidades virtuais (CV), comunidades virtual de aprendizagem (CVA), comunidades virtuais de interesse (CVI), comunidades virtuais de participação (CVP). Define-se, portanto nas próximas seções, estas expressões bem como a relação entre as mesmas a partir de estudos de vários autores (Wasserman e Faust, 1994; Rheingold, 1996; Wenger, 1998; Kilpatrick, et al., 2003; Palloff e Pratt, 2002; Palloff e Pratt, 2004; Illera, 2007; Recuero, 2009; Coll et al. 2010).

\section{Redes Sociais}

As redes sociais envolvem outros conceitos, como já mencionado na introdução. Nesse sentido, a figura 1 apresenta de maneira esquemática uma relação complexa e dinâmica, ou seja, uma interpretação das possíveis relações entre os significados dos elementos apresentados na referida figura. Ressalta-se que a conceituação e a descrição dos elementos da figura 1, neste artigo, não é linear devido à complexidade de cada um deles e devido as estruturas dinâmicas que os compõem. O dinamismo das relações é representado pelos contornos e pelas setas (Figura 1) mostrando, por exemplo, que podem existir comunidades nas redes sociais ou não; ou que uma CP pode ser também uma CA ou tornar-se uma CA e vice-versa. No decorrer do texto, outras relações são explicitadas e, além disso, a figura 1 será explorada em detalhe.

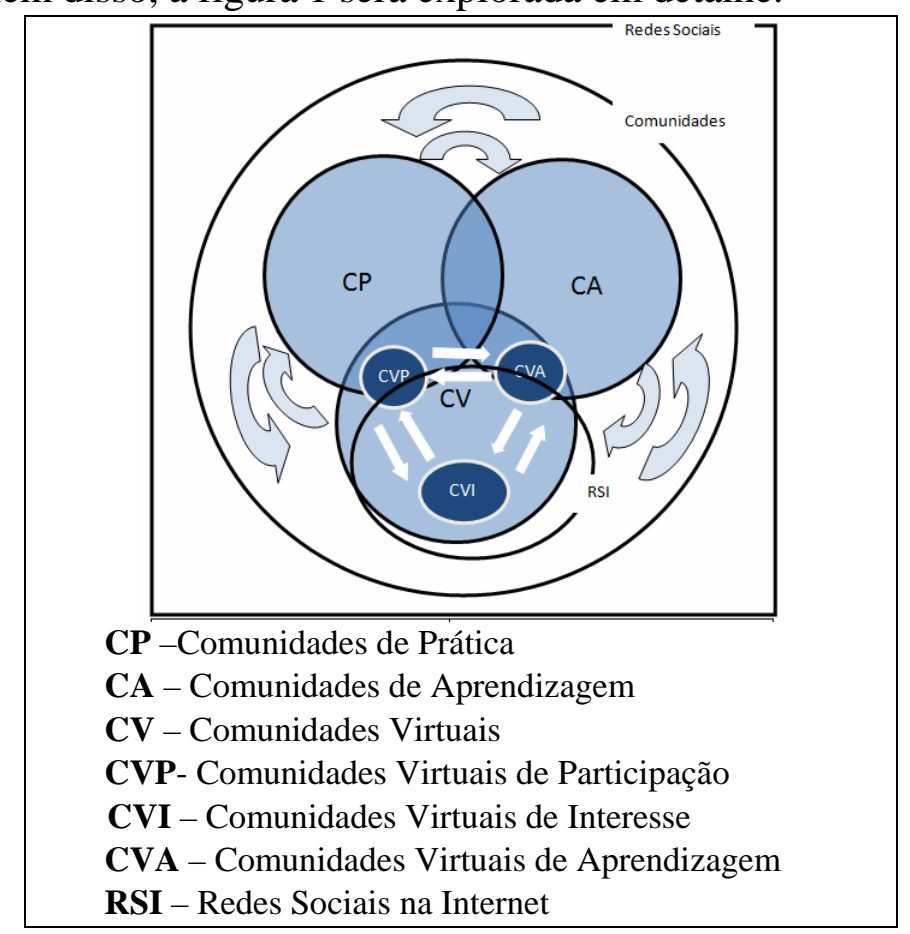

Figura 1 - Redes e Comunidades. Fonte: autora

Mesmo sendo "rede", um termo muito usado na literatura, sua caracterização tipológica e morfológica tem permanecido em aberto em vários trabalhos sobre o tema (Duarte et al., 2008). Não se pretende esgotar o assunto, mas sim apresentar algumas características e classificações, tanto de redes como de comunidades.

Uma rede social é definida como um conjunto de dois elementos: atores, ou seja, nós (pessoas, instituições ou grupos) e suas conexões (interações ou laços sociais) (Wasserman e Faust, 1994; Wellman, 1997). Capra (2008) complementa afirmando que redes sociais são redes de comunicação que envolvem linguagem simbólica, restrições culturais, relação de poder etc. Além disso, o referido autor afirma que são estruturas dinâmicas, complexas e autogenerativas ${ }^{1}$ e o que geram é imaterial. Enfim, as redes 
sociais por meio das interações vêm modificando diversas áreas da atividade humana, a saber: comércio, indústria, economia, artes, cultura e educação.

Os estudos sobre redes sociais não são novos, porém o enfoque deste modificou-se com o tempo. Até o século $\mathrm{XX}^{2}$, os cientistas estudavam as partes das redes, detalhadamente, visando, com isso, a compreender o todo. A partir do século XX iniciaram-se estudos com foco nas interações entre as partes (Recuero, 2009). Os estudos de Granovetter (2000) sobre laços sociais contribuíram para este novo foco, por meio da importância dada as interações. Tanto na parte social quanto nas outras ciências, o estudo de redes recebeu forte impulso após publicações de Barabási (2002), dentre outros autores, no final da década de 90.

O surgimento da Internet e em especial das ferramentas Web 2.0 possibilitaram a criação de espaços de troca virtuais, colocou em discussão na academia o conceito de territorialidade geográfica que era uma característica forte na sociologia até o momento. As redes, associadas às ferramentas da Web 2.0, possibilitam uma nova forma de relacionamento, independente de tempo e espaço, as chamadas redes sociais na Internet (RSI). Embora, segundo Castells (2003), o desaparecimento do lugar geográfico como forma de sociabilidade já existia antes do aparecimento da Internet. O uso de telefones, de cartas e de outros meios de comunicação possibilitou trocas comunicacionais independentes da localização.

Castells (2003) credita à estrutura da rede a ascensão do individualismo ("individualismo em rede"), justificando este fato na importância do papel do indivíduo na construção de sua própria rede social. Segundo o referido autor, na rede o indivíduo escolhe com quem irá interagir. "O individualismo em rede é um padrão social, não um acúmulo de indivíduos isolados. O que ocorre é que indivíduos montam suas redes, online e off-line, com base em seus interesses, valores, afinidades e projetos” (Castells, 2003, p.109). As formas de expressão numa rede social, como já mencionado, concretizam-se no estabelecimento de interações entre os nós da rede (atores da rede). Essas interações além de terem um caráter dinâmico permitem estabelecer os tipos de relacionamentos emergentes na rede e que comumente denominamos de laços sociais. Os laços podem ser fortes ou fracos de acordo com a qualidade das interações e das trocas sociais realizadas entre os atores. Laços fortes são aqueles que se caracterizam pela intimidade e pela proximidade, já os fracos caracterizam-se por relações esparsas, que não traduzem proximidade e intimidade (Wellman, 1997; Granovetter, 2000). Dessa forma, os laços fortes constituem as redes menos instáveis

As trocas que ocorrem nas redes sociais geram o capital social, esse é construído e negociado entre os atores e permite o aprofundamento dos laços e a sedimentação dos grupos (Recuero, 2009). Segundo Putnam (1993), capital social refere-se à conexão entre indivíduos, são normas e valores que governam interações entre as pessoas. Falk e Kilpatrick (2000) complementam afirmando que capital social são normas, valores e redes que podem ser usados para benefício mútuo. O "social” do capital social ressalta que estes recursos não são bens pessoais, estes residem em redes sociais. Essas definições são semelhantes e ao mesmo tempo complementares, sendo assim neste trabalho a definição de capital social adotada é a união das apresentadas. Para alguns, o capital social facilita a aprendizagem, promovendo a confiança, valores compartilhados, desenvolvimento pessoal, e acesso ao conhecimento de outras pessoas por meio das redes que formam uma base sólida para a partilha de conhecimentos e competências (Falk; Kilpatrick, 2000).

Segundo Recuero (2009), as RSI podem ser de dois tipos quanto à forma de integração: emergentes e de filiação (Recuero, 2009). As redes emergentes são aquelas cujas conexões entre os nós emergem por meio das trocas sociais realizadas pela 
interação social e pela conversação. Essas se caracterizam pela construção do grupo por meio da interação como, por exemplo, nos comentários de um weblog ou fotolog. Denomina-se emergente já que é constantemente construída e reconstruída pelas trocas sociais, que geralmente, são pequenas. Essas redes são mantidas pelo interesse dos atores em fazer amigos e dividir suporte social, confiança e reciprocidade (Recuero, 2009). O outro tipo de RSI, a de filiação (ou associação) é constituída por mecanismos de filiação de sites de redes sociais, é o caso, por exemplo, das listas de amigos no Orkut, da lista de pessoas que alguém segue no Twitter. Essas redes são mais estáveis e estáticas do que as emergentes e podem representar laços estabelecidos pelos atores em outros espaços. As redes de filiação não são alteradas pelo aumento ou diminuição de interações e de valores trocados, mas podem agregar valor à rede social e gerar capital social. Esse tipo de rede, geralmente, é grande, pois não é preciso interagir para manter a conexão, essa é mantida pelo sistema utilizado, sendo assim os laços podem ser fracos. De maneira geral, as diferenças entre as redes emergentes e as de filiação são sutis, nada impede, por exemplo, que uma rede de filiação crie laços fortes (Recuero, 2009).

Além de classificar as redes, destaca-se que um elemento importante para seu estudo é a compreensão dos grupos que são formados por meio das mesmas. As interações que ocorrem por meio da Internet estão possibilitando a formação de grupos sociais com características comunitárias. Segundo Wellman (2003), os laços sociais estão sendo ampliados por meio do desenvolvimento dos meios de comunicação e de transporte. Neste contexto, surge a comunidade virtual que, segundo Recuero (2009), é uma característica das redes sociais e a compõe. Apresentam-se na próxima seção diferentes tipos de comunidades que podem ser criadas numa rede social (Figura 1).

\section{Comunidades}

O conceito tradicional de comunidade tem sido modificado com o passar dos tempos, tem-se apontado para o caráter social das comunidades e o estabelecimento de relacionamentos como foco principal. Entre os principais autores que tratam deste tema, cita-se Wellman (1997). Este considera que comunidades são redes de laços interpessoais que proporcionam sociabilidade, apoio, integração e identidade social, entre outros aspectos. De maneira geral, comunidade pode ser entendida como um grupo de pessoas com características ou interesses comuns, que podem compartilhar um objetivo específico e que, geralmente, dividem um espaço geográfico (COLL et al., 2010). Para Illera (2007, p.118), “As comunidades, virtuais ou não, são sempre organizações temporais, coesas, mas multi-nível, tanto pelos interesses individuais (o tema em torno do qual gira a comunidade), como pelo enquadramento institucional e social em que ocorrem”. Participar de uma comunidade é um ato de geração mútua de autonomia, um meio pelo qual pessoas compartilham com outras, o que são e vivem colaborativamente, ou seja, não implica ignorar a individualidade ou submeter-se à determinada autoridade a fim de participar de um grupo (Palloff; Pratt, 2002).

Entre os diversos tipos de comunidades, nos últimos 15 anos, as expressões comunidade de aprendizagem (CA) e comunidades virtuais de aprendizagem (CVA) foram muito difundidas em diversas áreas, no contexto educacional, oraganizacional, político, cultural e empresarial (Coll et al., 2010). O interesse crescente pelas CA e CVA, segundo Coll, Bustos e Engel, (2010) está associado à: i) importância concedida ao conhecimento e à aprendizagem nas sociedades atuais; ii) a aceitação crescente de enfoques, pressupostos e teorias psicológicas e psicoeducacionais que destacam a importância dos fatores contextuais, sociais, culturais, relacionais e colaborativos; iii) o acelerado desenvolvimento das tecnologias de informação e comunicação (TIC), assim 
como sua ubiquidade e sua incorporação progressiva em praticamente todos os âmbitos da vida cotidiana; iv) a preocupação pela transformação e qualificação dos sistemas educacionais e das escolas. CA e CVA são conceitos que compartilham um núcleo básico de significados, mas apresentam diferenças. No final desta seção destacam-se algumas destas diferenças. Inicialmente, ressalta-se que nem toda comunidade é uma CA ou CVA ou mesmo uma comunidade de prática (CP).

Segundo Coll (2004), CA podem ser pensadas como espaços nos quais duas ou mais pessoas, com interesses comuns e relacionados com a aprendizagem, compartilham experiências, conhecimentos e expectativas diferenciadas num processo colaborativo trocando papéis e funções ao longo do tempo. As CVA apresentam estes mesmos princípios, porém as interações são via recursos da Internet.

As CP assim como, CA têm sido amplamente usadas em pesquisas de diversas áreas. As CP se referem a um grupo de pessoas que se unem em torno de um mesmo tema ou interesse, trabalham juntas para encontrar meios de melhorar o que fazem por meio de interações (Wenger, 1998). A sustentabilidade da CP não está na rede de relacionamentos, mas sim na sua identidade como comunidade e na identidade de seus membros por meio da prática compartilhada (Wenger, 1998). Os saberes de uma CP se expressam por meio das formas compartilhadas de fazer e entender no âmbito da comunidade, as quais resultam de dinâmicas de negociação envolvendo participação ativa e reificação ${ }^{3}$ da (Fiorentini, 2010). Participar de uma CP significa, portanto, engajar-se nas atividades da comunidade como membro atuante e produtivo.

A teorização sobre as CP pressupõe uma mudança nas concepções de aprendizagem. Essas comunidades se situam no contexto da instituição educativa, mais especificamente na própria vida das pessoas que aprendem (Illera, 2007). As CP não focam apenas na aprendizagem, mas na relação entre a aprendizagem e a vida pessoal e social. Esta característica revela a ligação deste tipo de comunidade com a teoria vygotskyana, na qual o contexto desempenha um papel importante. A aprendizagem na CP não tem um fim em si mesma (como ocorre nas abordagens meramente pedagógicas ou psicológicas), mas é uma componente a mais do conjunto de experiências (Illera, 2007).

As características determinantes de uma CP são: i) o campo de trabalho ou interesse; ii) o pertencimento, que implica um nível mínimo de conhecimento do campo; iii) interesse comum e não apenas ter o mesmo trabalho ou profissão; iv) a prática, afinal os membros desenvolvem um repertório compartilhado de recursos, experiências, histórias, ferramentas, formas de abordar e resolver problemas recorrentes. As CP, quando associadas a projetos educacionais, permitem aproximar a experiência escolar da vida cotidiana, embora nem sempre uma CP seja uma CA (Coll et al., 2010).

As tecnologias causaram mudanças nas redes, como já mencionado, o mesmo ocorreu com as comunidades, afinal possibilitaram a conquista de novos amigos e vivências de novas experiências sociais. Nesse contexto, surgem as comunidades virtuais (CV). Rheingold (1996) foi um dos primeiros autores a, efetivamente, utilizar a expressão $\mathrm{CV}$, para os grupos humanos que mantinham relações sociais no ciberespaço. Este as definiu como "agregados sociais surgidos na rede, quando os intervenientes de um debate o levam por diante em número e sentimento suficientes para formarem teias de relações pessoais no ciberespaço" (Rheingold, 1996, p.18). Ou seja, as comunidades virtuais são agregações virtuais que emergem na rede quando algumas pessoas se engajam em discussões públicas por certo tempo e com emoções para formar teias de relações pessoais na Internet (Rheingold, 1996). Assim, a diferença entre as CV e o restante da estrutura da rede social (Figura 1) não está nas pessoas, mas sim nos elementos de conexão (Recuero, 2009). Há muitas variações do conceito de CV, Hunter 
(2002), por exemplo, não ressalta, na sua definição de CV, o caráter virtual da comunidade, mas dá ênfase à participação dos membros

Nas CV há forte característica de agregação, estas comunidades possuem limite físico que é a capacidade de investimento dos atores sociais na manutenção das conexões da rede (Recuero, 2009). Nesse sentido, a CV constituir-se-á em um núcleo mais denso da RSI, contendo laços fortes e capital social (Recuero, 2009). Sempre há atores sendo incluídos na comunidade e outros saindo, gerando assim uma área média, "amigos dos amigos" que pode ser incluída na comunidade. Há também uma área externa, constituída pelo restante da rede, laços fracos e com menos capital social construído. Nesta idéia, associa-se a noção de comunidade ao núcleo da maioria dos grupos sociais (Recuero, 2009). No núcleo (comunidade) há comprometimento, organização e predominância dos laços fortes.

As redes sociais, de maneira geral, são mais fluidas que as comunidades, essas últimas necessitam da predominância de interações cooperativas, para gerar e manter sua estrutura de comunidade (Recuero, 2009). Sendo assim, as CV se distinguem das RSI, pelo fato de que nas primeiras são observados laços fortes que formam grupos sólidos, há colaboração entre os integrantes, alto grau de adaptação, auto-organização e sincronismo (Carvalho, 2009). Uma CV pode situar-se dentro de uma RSI (Figura 1), mas também pode surgir como comunidade, além disso, é possível que uma RSI se transforme numa CV (Carvalho, 2009), tudo isto representa a dinamicidade do processo de formação das redes e das comunidades. As CA e as CP entraram na era digital, podem ser virtuais (Ilerra, 2007), o que altera as formas de mediação e interação agregando assim novas características às mesmas.

A organização, a estrutura e a gestão das CV se caracterizam por (Shumar; Renninger, 2002): i) não possuem localização temporal nem espacial, sendo assim são mais intencionais e simbólicas do que as comunidades físicas; ii) as relações se definem por interesse e não somente por proximidade; iii) o tempo de interação pode ser expandido ou comprimido; iv) o espaço da CV permite ampliar o tipo, a forma e o volume dos recursos; v) as ideias apresentadas e compartilhadas pelos participantes podem ser acumuladas.

Segundo Coll, Bustos e Engel (2010) podem-se determinar três níveis de benefícios associados aos objetivos e expectativas dos membros das CV. No primeiro nível, o objetivo surge do interesse por um tema, um acontecimento ou um fenômeno, constituindo assim comunidades virtuais de interesse (CVI). Essas comunidades permitem que seus membros obtenham informação atualizada sobre o objeto de interesse a qualquer hora de qualquer lugar. Os membros filiam-se voluntariamente e comunicam-se com outros que podem ou não compartilhar pontos de vistas. As CVI, geralmente, são grandes, formadas por centenas ou milhares de pessoas que trocam informações sobre o objeto de interesse, mas não aspiram, necessariamente, envolver-se em um processo de aprendizagem (Coll et al., 2010).

No segundo nível estão as comunidades virtuais de participação $\left(\mathrm{CVP}^{4}\right)$, nestas o objetivo dos membros é participar, envolver-se. O interesse pode ser fator inicial para participação, mas neste nível há um sentido de responsabilidade coletiva na busca de melhores práticas, na solução coletiva de problemas, na discussão de questões complexas ou na análise de situações e propostas apresentadas pelos membros da comunidade. A filiação, geralmente, é voluntária, embora possa ser requerida como ocorre, por exemplo, nas CVP criadas em organizações ou empresas (Coll et al., 2010).

No terceiro nível estão as CV que têm como objetivo explícito a aprendizagem e seus membros desenvolvem estratégias, planos, atividades e papéis específicos para atingir um objetivo, são as comunidades virtuais de aprendizagem (CVA). Nessas a 
filiação também pode ser voluntária, mas, geralmente, nos casos institucionais são criadas para que os membros se integrem e participem como parte de seus programas regulares, ou seja, filiação não voluntária. Neste caso, a participação e o compromisso são controlados pelas instituições ou programas educacionais (Coll et al., 2010).

Segundo Coll (2004), as CVA têm como característica principal a construção coletiva do conhecimento. Sartori e Roesler (2003) complementam afirmando que as CVA são criadas a partir de objetivos definidos, visando principalmente ao desenvolvimento de habilidades e competências focadas na formação geral ou profissional de determinado grupo. Estas congregam pessoas e oferecem recursos, permitindo a busca de informações e a comunicação, com o objetivo comum de aprender. A sensação de pertencimento, de fazer parte de um grupo que interage e aprende conjuntamente, traz mais confiança e favorece a aprendizagem.

Palloff e Pratt (2002) complementam afirmando que para diversos autores uma CVA se constrói a partir dos seguintes passos: i) definir claramente a proposta do grupo; ii) criar um local diferenciado para o grupo; iii) promover lideranças internas eficientes; iv) definir normas e um claro código de conduta; v) permitir que haja uma variedade de papéis para os membros do grupo; vi) permitir e facilitar subgrupos; vii) permitir que os participantes resolvam suas próprias discussões.

Os indicadores de que uma CVA está sendo formada segundo Palloff e Pratt (2002), são: i) interação ativa, envolvendo tanto o conteúdo de um curso quanto a comunicação pessoal; ii) aprendizagem colaborativa, observada pelos comentários realizados entre os alunos e entre o professor e o aluno; iii) compartilhamento de recursos entre os alunos; iv) expressões de apoio e de estímulo trocadas entre alunos. Um caminho para identificar uma CVA é observar se as interações revelam os referidos indicadores.

As fronteiras entre os três níveis de CV (CVI, CVP e CVA) não são delimitadas com exatidão, elas são dinâmicas. Além disso, são expostas a contínuos processos de mudança e transformação de modo que uma CVI pode evoluir, transformando-se em uma CVP ou mesmo numa CVA, isto pode ocorrer no sentido contrário.

O que difere as CVA das demais comunidades virtuais é o envolvimento com a aprendizagem colaborativa e prática reflexiva implícita na aprendizagem transformadora (Palloff; Pratt, 2004). Já o que difere a CVA das CA é que a primeira é constituída por meio da Internet, porém é importante destacar que uma CA pode se tornar uma CVA e vice-versa, o mesmo pode acontecer com as CP. A constituição e a manuntenção das comunidades são processos dinâmicos, essas podem, inclusive deixar de existir.

De maneira geral, é importante que as comunidades estejam organizadas sobre a base de um interesse comum de criar e recriar o conhecimento, além de compartilhá-lo (Imbernón, 2010). Tudo isso pode diversificar e enriquecer os conhecimentos, ou até gerar novos. Segundo Moreira e Loureiro (2009), as comunidades podem apoiar a aprendizagem de professores, minimizando problemas como sustentabilidade dos programas de formação, a imersão no trabalho diário dos professores dos seus programas de desenvolvimento profissional e o desenvolvimento do professor enquanto investigador.

\section{Sites de Redes Sociais}

Para a criação de RSI e de CV existem muitas plataformas ${ }^{5}$ disponíveis na Internet, a saber: Orkut, Ning, SocialGO, Meezoog, WackWall, Grouply, Peabirus, Elgg, entre outras. Recuero (2009) denomina essas plataformas de sites de redes sociais (SRS) ${ }^{6}$, ou seja, são espaços utilizados para a expressão das redes sociais e comunidades na Internet. Geralmente, essas ferramentas são usadas para fazer amigos, compartilhar fotos, vídeos e comentários. Mas, podem representar importante recurso para o processo 
de ensino e aprendizagem, podem dar suporte formal e informal à modalidade presencial ou a distância. A referida autora afirma que:

Embora os sites de redes sociais atuem como suporte para as interações que constituirão as redes sociais, eles não são, por si, redes sociais. Eles podem apresentá-las, auxiliar a percebê-las, mas é importante salientar que são, em si, apenas sistemas. São os atores sociais, que utilizam essas redes, que constituem essas redes (RECUERO, 2009, p. 103).

Alguns SRS permitem maior controle do criador, são aqueles que requerem um servidor no qual é criado o domínio. Para fins educacionais possuir um domínio é de suma importância, pois mesmo os SRS gratuitos trazem riscos para os usuários, visto que podem desaparecer da web ou deixar de oferecer os serviços gratuitamente a qualquer momento. Caso isso aconteca, todo conteúdo hospedado no SRS pode ser perdido, gerando prejuízos a manutenção das redes e das comunidades ${ }^{7}$.

Podem-se classificar os SRS em dois tipos: os SRS propriamente ditos e SRS apropriados (Recuero, 2009). Os SRS propriamente ditos são sistemas focados em expor e publicar as redes sociais dos atores (Orkut, facebook, Ning, Elgg e outros). Já os SRS apropriados são sistemas que não foram criados, originalmente, para mostrar redes sociais, mas que são apropriados pelos atores com este fim (Fotolg, weblogs, Twiter e outros). Nestes sites, diferentemente do outro tipo, não há espaço para específicos para perfil e para publicização das conexões (Recuero, 2009). O perfil é construído por meio de espaços pessoais ou perfil pela apropriação dos atores. È importante destacar que os SRS não representam redes independentes entre si, geralmente um mesmo ator utiliza diversos SRS com diferentes objetivos. Segundo Coll, Bustos e Engel (2010), uma plataforma ou SRS adequado é aquele que proporciona à comunidade os serviços básicos para proporcionar a geração dos processos de comunicação e interação social necessário para se alcançar os objetivos estabelecidos.

\section{Considerações Finais}

As redes sociais e os diversos tipos de comunidades, na Internet ou não, podem contribuir para a formação inicial ou continuada de professores, por meio das interações que possibilitam a criação de laços sociais e a criação de capital social.

Diante de todo o aporte teórico apresentado neste artigo considera-se que uma rede social pode contribuir para o desenvolvimento de uma CP (na Internet ou não). As características das redes sociais e das CP sinalizam que estas são importantes recursos para apoiar um modelo de formação continuada. Esta afirmação não exclui o uso de outros tipos de comunidades descritos, para o mesmo fim.

Estes conceitos serão utilizados na análise e validação de um modelo de formação continuada cujo objetivo será contribuir para integração das TIC às práticas docentes de professores inciantes de Matemática (Barcelos, 2010). Este modelo contemplará o uso de redes sociais e CP, para tanto entre outros recursos, pretende-se usar a plataforma Elgg. O foco da CP estará nas interações, em particular nas mediações, por meio de instrumentos (tecnologias) e de outro indivíduo mais experiente. Além disso, o contexto do professor em formação será considerado no desenvolvimento das atividades. A pesquisa para elaboração do modelo ainda não foi concluída, está sendo realizado um estudo de implementação por meio de um estudo de caso. Com a criação do modelo de formação continuada buscam-se mudanças na prática docente.

\section{Notas de Texto}

\footnotetext{
${ }^{1}$ As redes são criadas e recriadas, transformando ou substituindo seus componentes (Capra, 2008).

${ }^{2}$ A teoria de redes de Erdos e Rényi dominou o pensamento científico sobre as redes desde a sua introdução em 1959 (Barbási, 2002).
} 


\footnotetext{
${ }^{3}$ Para Wenger (1998), reificação significa “Tornar em coisa”, o que não significa necessariamente algo concreto ou material, refere-se ao processo de dar forma e sentido à experiência humana mediante produção de objetos tais como artefatos, idéias, conceito ou textos escritos.

${ }^{4}$ Este termo não equivale as $\mathrm{CP}$ ou mesmo as comunidades virtuais de prática (Coll et al., 2010), as CP possuem características próprias, como já foi apresentada nesta seção.

${ }^{5}$ Também denominadas ferramentas, sistemas, sites ou softwares.

${ }^{6}$ Boyd e Ellison (2007) afirmam que SRS são aqueles que permitem: i) a construção de um perfil ou página pessoal; ii) a interação por meio de comentários; e iii) ver e percorrer a sua lista de conexões e aquelas feitas por outras pessoas dentro do sistema.

${ }^{7}$ Isto aconteceu com a plataforma Ning, a partir do dia 4 de Junho de 2010 este SRS deixa de oferecer seus serviços gratuitamente.
}

\section{Referências Bibliográficas}

BARABÁSI, A. L. Linked: The new science of networks. Cambridge, Massachusetts: Perseus Publishing, 2002.

BARCELOS, G. T. Modelo de formação continuada: integração das tecnologias de informação e comunicação à prática docente de professores iniciantes de matemática. Proposta de Tese. Programa de Pós Graduação em Informática na Educação, UFRS, Porto Alegre, RS, 2010.

BOYD, D. M.; ELLISON, N. B. Social network sites: Definition, history, and scholarship. Journal of Computer-Mediated Communication, v.13, n.1, article 11, 2007.

CAPRA, F. Vivendo Redes. In: DUARTE, F.; QUANDT, C.; SOUZA, Q. (org.) O tempo das redes. São Paulo: Perspectiva, 2008. p.17-29.

CARVALHO, J. de S. Redes e Comunidades virtuais de aprendizagem: elementos para uma distinção. São Paulo, SP, Faculdade de Educação da Universidade de São Paulo, 2009. Dissertação de Mestrado em Educação.

CASTELLS, M. A Galáxia na Internet: reflexões sobre a Internet, os negócios e a sociedade.Tradução de Maria Luíza X. de A. Borges. Rio de Janeiro: Jorge Zahar, 2003.

COLL, C. Las Comunidades de Aprendizaje: nuevos horizontes para la investigación en psicología de la educatión. In: CONGRESSO INTERNACIONAL DE PSICOLOGÍA Y EDUCACIÓN. Simpósio Nuevos horizontes en Psicologia de la Educación, 2004, España Disponível em: <http://www.ub.es/grintie/GRINTIE/Library/public/CC_Almeria_04.pdf>. Acesso em: 15 abr. 2010.

COLL, C.; BUSTOS, A.; ENGEL, A. As comunidades virtuais de aprendizagem. In: COLL, C.; MONEREO, C e colaboradores. Psicologia da Educação Virtual: aprender e ensinar com as tecnologias de informação e da comunicação. Tradução de Naila Freitas. Porto Alegre: Artmed, 2010, p. 268-286.

DUARTE, F.; QUANDT, C.; SOUZA, Q. (org.) O tempo das redes. São Paulo: Perspectiva, 2008.

FALK, I.; KILPATRICK, S. What is social capital? A study of rural communities. Sociologia Ruralis, v.40, n. 1, p. 87-110, 2000.

FIORENTINI, D. Desenvolvimento profissional e comunidades investigativas. In: CUNHA, A. M. de O. et al. Convergências e tensões no campo da formação e do trabalho docente. Belo Horizonte, MG:Autêntica, 2010, p.570 -590.

GRANOVETTER M. La fuerza de los vínculos débiles. Tradução de Maria Ángeles García Verdasco. Política y Sociedad, Madrid. n. 33, p. 41-56, 2000.

HUNTER, B. Learning in the Virtual Community Depends upon Changes in Local Communities. In: RENNINGER, A. SHUMAR, W. (eds). Building Virtual 
Communities: learning and change in Cyberspace. New York: Cambridge University, 2002, p.96-126.

ILLERA, J. L. R. Como as comunidades virtuais de prática e de aprendizagem podem transformar a nossa concepção de educação. Revista de Ciências da Educação. n.3 p.117-124, 2007.

IMBERNÓN, F. Formação Continuada de Professores. Tradução de Juliana dos Santos Padilha. Porto Alegre: Artmed, 2010.

KILPATRICK, S.; BARRETT M.; JONES, T. Defining Learning Communities. Centre for Research \& Learning for in Regional Australia (CRLRA) Discussion Paper Series, D1/2003. Launceston: University of Tasmania. Disponível em: <www.crlra.utas.edu.au/files/discussion/2003/D1-2003.pdf > Acesso em 01 maio 2010.

MOREIRA, A.; LOUREIRO, M. J. Enquadramento das TIC na Formação Contínua de Professores. In: COSTA, F. (Coord.). Competências TIC. Estudo de Implementação, v.2 . Lisboa: GEPE/ME (Gabinete de Estatística e Planejamento da Educação), 2009. p 117-160. Disponível em: < http://www.pte.gov.pt/pte/PT/Projectos/Projecto/Documentos/index.htm?proj=47> Acesso em: 15 jan. 2010.

PALLOFF, R. M.; PRATT, K.. O Aluno Virtual: um guia para trabalhar com estudantes on-line. Tradução de Vinicius Figueira. Porto Alegre: Artmed, 2004.

PALLOFF R. M.; PRATT, K. Construindo Comunidades de Aprendizagem no Ciberespaço: estratégias eficientes para salas de aula on-line. Tradução de Vinicius Figueira. Porto Alegre: Artmed, 2002.

PUTNAM, R. Making democracy work: civic traditions in modem Italy. Princeton: Princeton University Press, 1993.

RECUERO, R. Redes Sociais na Internet. Porto Alegre: Sulina, 2009.

RHEINGOLD, H. A Comunidade Virtual. Lisboa: Editora Gradiva, 1996.

SARTORI, A.S.; ROESLER, J. Comunidades Virtuais de Aprendizagem: Espaços de Desenvolvimento de Sociedades, Comunicação e Cultura. In: Simpósio E-agora, professor? Para onde Vamos? São Paulo, 2003. Disponível em: $<$ http://www.pucsp.br/tead/n1a/artigos\%20pdf/artigo1.pdf>. Acessoem:16 de abr. 2010. SHUMAR, W.; RENNINGER, A. On Conceptualizing Community In: RENNINGER, A. SHUMAR, W. (eds). Building Virtual Communities: learning and change in Cyberspace. New York: Cambridge University, 2002, p.1-17.

TARDIF, M.; RAYMOND, D. Saberes, tempo e aprendizagem do trabalho no magistério. Educação \& Sociedade, Campinas, 2000, v.21, n.73, p. 209-244. Disponível em: <http://www.scielo.br/pdf/es/v21n73/4214.pdf>Acesso em:10fev. 2010.

WASSERMAN, S.; FAUST, K. Social Network Analysis: methods and applications. Cambridge, UK: Cambridge University Press, 1994.

WELLMAN, B. An Electronic Group is Virtually a Social Network. In: KIESLER, Sarah (org.) Culture of Internet. Hilsdale, NJ: Lawrence Erlbaum, 1997. p. 179-205.

WELLMAN, B. et. al. The Social Affordances of Internet for Networked Individualism. In: Journal of computer Mediated Communication, v. 8, Issue 3, 2003. Disponível em: <http://jcmc.indiana.edu/vol8/issue3/wellman.html>Acessoem:05 de maio de 2010. WENGER, E. Communities of Practice: Learning, Meaning, and Identity. Cambridge, CUP, 1998. 